\title{
Green shooting: análisis del discurso en la prensa de la producción audiovisual sostenible en España (2015-2019)
}

\section{Green shooting: analysis of the discourse in the press of sustainable audiovisual production in Spain (2015-2019) \\ Green shooting: análise do discurso na imprensa da produção audiovisual sustentável na Espanha (2015-2019)}

Vanessa Roger-Monzó, ESIC Business \& Marketing School, ESIC University, Madrid, España (vanessa.roger@esic.edu)

\begin{abstract}
RESUMEN | El objetivo de este estudio es analizar el tratamiento de la prensa escrita sobre la producción audiovisual sostenible en España. Se realiza un análisis cuantitativo y textual de las noticias aparecidas en medios nacionales y en lengua española sobre producciones sostenibles entre 2015 y 2019. Se obtienen 108 registros. El análisis del corpus lingüístico se realiza mediante un software de análisis textual.Los resultados sugieren que las principales temáticas informativas se asocian, principalmente, a propuestas para reducir el impacto medioambiental de estas producciones, celebración de festivales de cine sostenible, utilización de obras audiovisuales para educar en valores ecológicos y divulgación de tendencias sostenibles en el sector audiovisual a nivel europeo.
\end{abstract}

PALABRAS CLAVE: Green shooting; Green filming; objetivos de desarrollo sostenible; audiovisual; sostenibilidad. 
ABSTRACT/The aim of this studyis to analyze the treatment made by the press on sustainable audiovisual production in Spain. We conducted a quantitative and textual analysis of the news that appeared in national media and in Spanish on sustainable productions between 2015 and 2019. 108 news are obtained. The analysis of the linguistic corpus was made using textual analysis software. The results suggest that the main topics addressed are mainly linked to proposals to reduce the environmental impact of these productions, the celebration of sustainable film festivals, the use of audiovisual works to educate on ecological values, and the dissemination of sustainable trends in the audiovisual sector at European level.

KEYWORDS: Green shooting; Green filming; sustainable development goals; audiovisual; sustainability.

RESUMO|O objetivo deste estudo é analisar o tratamento da imprensa escrita sobre a produção audiovisual sustentável na Espanha. É realizada uma análise quantitativa e textual das notícias veiculadas na mídia nacional e em espanhol sobre produções sustentáveis entre 2015 e 2019. Foram obtidos 108 registros. A análise do corpus linguístico é realizada por meio de um software de análise textual. Os resultados sugerem que os principais tópicos informativos estão associados, principalmente, a propostas de redução do impacto ambiental destas produções, realizando festivais de cinema sustentáveis, utilizando obras audiovisuais para educar sobre os valores ecológicos e divulgando tendências sustentáveis no sector audiovisual.

PALABRAS CLAVE: green shooting; green filming; objetivos de desenvolvimento sustentável; audiovisual; sustentabilidade. 


\section{INTRODUCCIÓN}

El desarrollo sostenible se presenta como un nuevo modelo en el que convergen tres perspectivas: económica, social y medioambiental (Brundtland, 1987; Van Kleef \& Roome, 2007; Fernandes et al., 2019). Durante los últimos años, la preocupación por el medioambiente ha evolucionado y se ha extendido a la sociedad. Existe una mayor conciencia social de los problemas de sostenibilidad (Moore, 2005) y se ha forjado un fuerte proceso de transición sostenible (Markard et al., 2012; Geels, 2010). En España, según el Centro de Investigaciones Sociológicas, desde 2014 la inquietud ciudadana por el medioambiente y la sostenibilidad se ha triplicado (CIS, 2019).

Para alcanzar el desarrollo mundial sostenible, en septiembre de 2015 los estados miembros de las Naciones Unidas aprobaron el marco global Transformar nuestro mundo: la Agenda 2030 para el Desarrollo Sostenible (https://www.undp.org/ sustainable-development-goals), acuerdo que entró en vigor el 1 de enero de 2016. Esta agenda está integrada por 17 Objetivos de Desarrollo Sostenible (ODS) que conforman la hoja de ruta universal para erradicar la pobreza y la desigualdad, luchar contra el cambio climático, la degradación ambiental y fomentar la paz y la justicia, entre otros aspectos.

Por otra parte, los temas ambientales son reconocidos como puntales esenciales en estrategias de innovación de las diferentes industrias (Noci \& Verganti, 1999; Orlitzky et al., 2011), permiten diseñar nuevos modelos de negocio (Stubbs \& Cocklin, 2008; Joyce \& Paquin, 2016) y establecer cambios positivos a largo plazo (AragónCorrea et al., 2008). Buscar tecnologías y prácticas empresariales sostenibles en la industria audiovisual puede identificarse como una oportunidad para reducir el excesivo consumo energético de estas producciones y las innovaciones derivadas podrían aplicarse a otras industrias (Victory, 2015).

En este contexto, es necesario desplegar una comunicación para la sostenibilidad (Carpentier \& Servaes, 2006; Barker, 2007) dirigida a favorecer la toma de conciencia acerca de la realidad que transmiten los medios de comunicación (DíazNosty, 2009). Siguiendo el concepto de realidad refractada de Lippmann (2003), los medios proporcionan a los ciudadanos la información que integra diferentes versiones de la realidad. De este modo, desempeñan un rol fundamental en los marcos de referencia que se utilizan para interpretar temas de interés público (Scheufele, 1999), siendo esenciales para estructurar el tejido social y configurar la opinión pública (McQuail, 2000).

Elobjetivo de este análisis es examinar la evolución de la representación mediática sobre la producción audiovisual sostenible, identificar el tratamiento que realizan los medios de comunicación españoles sobre este tema, y determinar qué prácticas sostenibles se están desarrollando desde la industria audiovisual en España. 


\section{La comunicación de lo sostenible en la producción audiovisual}

Los medios constituyen un sistema interpretativo central de las sociedades modernas (Schmidt et al., 2013). En el proceso de socialización de la sostenibilidad construyen una imagen pública de estos aspectos (Fernández-Reyes, 2004). Las audiencias demandan contenidos que profundicen en las causas y consecuencias de los hechos. La especialización periodística da respuesta a las necesidades de los usuarios y brinda información de calidad (Fernández del Moral \& Esteve, 1993). Surgen así el periodismo sostenible, que integra el periodismo tecnocientífico vinculado con la información que genera la ciencia y la tecnología, el periodismo energético, que aborda modelos energéticos, y el periodismo ecopolítico, enfocado a temas ambientales desde una perspectiva política (Fernández-Reyes, 2004).

El proceso de realización de obras audiovisuales a menudo se considera limpio y generalmente benigno (Flanigan, 2002). Sin embargo, las producciones cinematográficas, televisivas y publicitarias causan un gran impacto medioambiental, debido fundamentalmente al suministro de agua, utilización de envases plásticos, uso del transporte y construcción de decorados, entreotros aspectos (Corbett\&Turco, 2006).

Diversos estudios confirman el elevado nivel de contaminación que genera una producción audiovisual (Victory, 2015; Corbett \& Turco, 2006). En los últimos años ha surgido una nueva tendencia, denominada green shooting o green filming, dirigida a reducir la energía, los recursos empleados y a buscar alternativas sostenibles durante la preproducción, la producción y la posproducción. Se impulsan medidas como, por ejemplo, fomentar tecnologías que ahorren energía, limitar el desperdicio y la utilización excesiva de materiales de un único uso, eliminar botellas plásticas de agua y cambiarlas por fuentes portátiles, usar vehículos eléctricos y consumir catering de comida local, impulsando así el comercio local (Lopera-Mármol \& Jiménez-Morales, 2021). Se han implementado diversos protocolos para la producción cinematográfica sostenible en todo el mundo, especialmente en países anglosajones (Chiarini \& Khedachi, 2019). De hecho, Producer's Guild of America detalla en su Guía de Producción Ecológica las mejores prácticas para la producción audiovisual sostenible, ofreciendo una calculadora de huella de carbono para evaluar su impacto ambiental (Clevé, 2017). En Europa existen iniciativas similares, lo que da cuenta de la importancia que se otorga a la vertiente medioambiental del desarrollo sostenible en la industria (Lopera-Mármol \& Jiménez-Morales, 2021).

Sin embargo, las regulaciones y diferencias culturales y logísticas en cada país complican la implementación de estas medidas, y existe una gran dificultad para unificar dichos protocolos bajo una marca registrada (O'Brien, 2014). En España, la adopción del green shooting es incipiente en el sector audiovisual y las iniciativas que impulsan políticas generales de sostenibilidad en este ámbito todavía son reducidas. 
Por otra parte, la visibilidad de esta industria, en parte debido a la influencia de sus agentes (directores, actores, etc.), podría aprovecharse para convertir el sector en un catalizador orientado a un cambio sostenible (Chiarini \& Khedachi, 2019), puesto que las entidades preocupadas por su reputación y deseosas de mejorar su imagen corporativa apuestan por obtener mayor visibilidad en medios (Castelo \& Lima Rodrigues, 2008). Así pues, los medios de comunicación constituyen una herramienta útil para que los agentes implicados puedan transmitir sus iniciativas e influir en la opinión pública (Schweinsberg, Darcy, \& Cheng, 2017).

\section{Objetivos de la investigación}

Los objetivos de esta investigación son examinar la evolución de la representación mediática de las noticias relativas a la producción audiovisual sostenible en la prensa española, para comprobar el interés de los medios por este tema, e identificar en dichas noticias las principales iniciativas vinculadas con los ODS de la Agenda 2030 impulsadas desde diversas entidades y que pueden servir como guías para estructurar el diseño de las políticas de sostenibilidad y configuración de las transiciones sociotécnicas en este sector. De este modo, será posible identificar y analizar el repertorio de opciones estratégicas disponibles para la industria audiovisual con el objetivo de contribuir a su desarrollo sostenible.

\section{METOdOLOGÍA}

Se seleccionaron las noticias publicadas en la prensa española sobre este tema entre 2015 y 2019. Dicho periodo incluye la aprobación de la Agenda 2030 de Naciones Unidas (septiembre de 2015) y su entrada en vigor (1 de enero de 2016).

En primer lugar, se examina la evolución del número de noticias publicadas en medios nacionales y en lengua española que incluyen referencias a las producciones audiovisuales sostenibles. Asimismo, se realiza un análisis textual o semántico de tipo cualitativo-cuantitativo de estas.

Para explorar las publicaciones se empleó Factiva, una base de datos de información que permite consultar 264 medios nacionales.

La búsqueda comprende las diferentes variantes de los términos rodaje sostenible, audiovisual sostenible, cine sostenible, green filming y green shooting que aparecen en el titular de la noticia, ya que según Núñez Ladevéze (1995) los titulares concentran la principal idea de la noticia presentada de forma atractiva y sintetizada.

Inicialmente, se recuperan 125 resultados que se examinan con el fin de escoger aquellos en los que la producción audiovisual sostenible se aborda de forma relevante. Se descartan 17 informaciones, por lo que la muestra final engloba 108 registros. 
El análisis textual de las 108 noticias objeto de estudio se lleva a cabo a través del software T-LAB versión Plus 2020. Se trata de una aplicación que engloba herramientas estadísticas, gráficas y lingüísticas para examinar textos mediante la identificación de patrones de palabras. El proceso de análisis de T-LAB se sustenta en dos categorías diferenciadas: los contextos elementales y las unidades lexicales.

Los contextos elementales son el resultado de la segmentación del corpus lingüístico para efectuar el análisis del cómputo de coocurrencias. En rigor, se trata de proporciones del texto del corpus que se corresponden con unidades sintagmáticas de una o más frases o párrafos de las diferentes noticias seleccionadas.

Las unidades lexicales contienen dos informaciones: palabra y lema. La palabra se muestra tal y como aparece en el corpus, mientras que el lema constituye la etiqueta atribuida a las unidades lexicales agrupadas y clasificadas según diccionarios personalizados, categorías semánticas que engloban términos con el mismo significado para la investigación o criterios lingüísticos. Este proceso se denomina lematización. Por ejemplo, los términos sostenible y sostenibilidad se han agrupado bajo una misma etiqueta (lema) -sostenible-, al considerar que tienen el mismo significado para la investigación.

La normalización automática inicial que proporciona T-LAB arroja 526 unidades lexicales. Después de renombrar y reubicar los lemas disponibles, se identificaron 48 unidades lexicales. Para garantizar la fiabilidad de los datos estadísticos durante la fase preprocesamiento, T-LAB calcula un umbral de frecuencia en la selección de los lemas que, en esta ocasión, se ha situado en 10.

A partir de las 48 unidades lexicales establecidas, se han realizado diversos análisis de coocurrencias, es decir, los resultados derivados de calcular el número de veces que dos o más unidades lexicales coinciden en los mismos contextos elementales. De este modo, es posible determinar el discurso de los medios sobre este tema. Específicamente, se ha establecido un análisis de asociaciones de lemas y un análisis de comparaciones entre parejas de lemas. Asimismo, se ha elaborado una clasificación de clústeres para obtener una representación de los contenidos del corpus mediante grupos temáticos significativos, la que permite determinar los ámbitos que se abordan cuando se hace referencia a producciones sostenibles.

\section{RESULTADOS}

\section{Análisis de la representación mediática}

El análisis descriptivo de la evolución de la representación mediática de noticias publicadas sobre el tema objeto de estudio se ilustra en el gráfico 1. La presencia de las noticias sobre la producción audiovisual sostenible en la prensa analizada crece a lo largo de los años. 


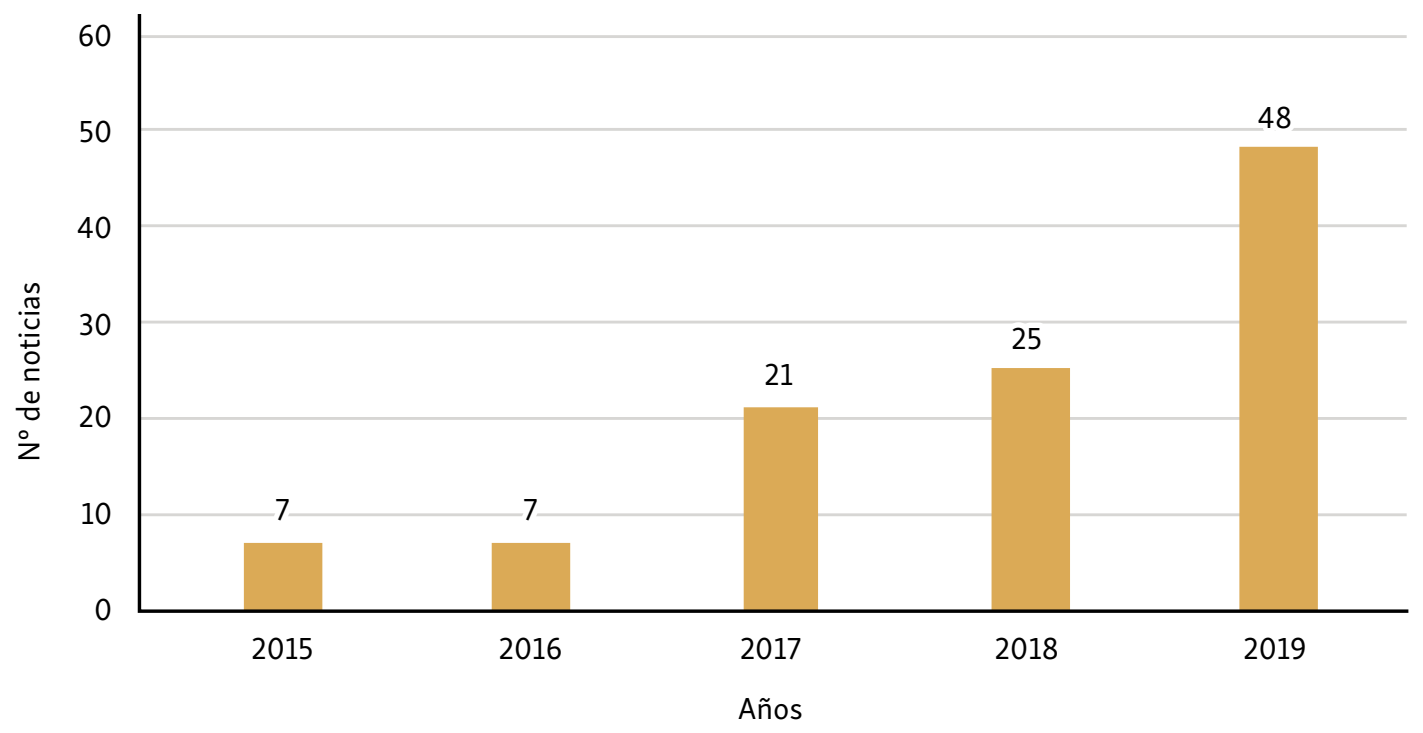

Gráfico 1. Número de noticias sobre el audiovisual sostenible por año

Fuente: Elaboración propia.

Fuente de información

Agencia Europa Press

Agencia EFE

Prensa nacional (cabeceras)
Número de noticias

45

12

41

Tabla 1. Número de noticias publicadas por tipo de medio

Fuente: Elaboración propia a partir de datos de Factiva.

Se observa que durante los años 2015 y 2016 la presencia de publicaciones que abordan la temática de producción sostenible de obras audiovisuales es incipiente, con 7 publicaciones para cada año. Esta cifra se explica porque la Agenda 2030 se aprobó a finales de 2015 y se inició el 1 de enero de 2016. El incremento de este tipo de informaciones se da a partir de 2017 (21 noticias), con un ligero incremento en 2018 ( 25 noticias). En 2019 prácticamente se duplica el número (48 noticias) con respecto del año anterior, y la sostenibilidad relacionada con el contexto audiovisual adquiere mayor presencia en la agenda mediática. Estos datos coinciden con el incremento de la concienciación ciudadana por el medioambiente y la sostenibilidad que, desde 2014, se ha triplicado (CIS, 2019).

La tabla 1 muestra los medios que publicaron noticias sobre rodajes sostenibles. 
La agencia de noticias Europa Press, seguida muy de lejos por la agencia EFE proveedoras de contenido de un gran número de medios de comunicación (Johnston \& Forde, 2011), especialmente regionales (Artero \& Moraes, 2008)- son las fuentes de información que elaboran mayor número de noticias sobre esta temática. Así, actúan como vehículo de divulgación de este tema a la opinión pública, siendo el primer medio de transmisión sobre las transiciones sociotécnicas del sector audiovisual. Destaca una gran atomización en torno a las cabeceras de prensa nacional. A pesar de que la sostenibilidad en la producción audiovisual es un tema que en los últimos años ha penetrado en los medios (Lopera-Mármol \& JiménezMorales, 2021), todavía no es un aspecto generalizado.

\section{Análisis del discurso}

Identificación de principales lemas

El gráfico 2 muestra la distribución de los lemas más frecuentes en las 108 noticias que constituyen el corpus lingüístico analizado. Se establece un umbral de 50 apariciones.

Se observa que, después del lema sostenible, los más repetidos se vinculan con:

- La industria cinematográfica (cine, rodaje, película, documental).

- Certámenes y encuentros (festival, jornadas).

- Acciones y efectos medioambientales (medioambiente, medidas, reducir, impacto, cambio climático).

- Sectores empresariales (cultura, turismo, empresas, audiovisual, producción).

De estos datos se desprende que la sostenibilidad define las empresas de diversos sectores, entre los que se encuentra la industria audiovisual, cuyas principales prácticas sostenibles se vinculan con los ODS relacionados con aspectos ecológicos (medioambiente, cambio climático), tal y como lo señalan Lopera-Mármol y Jiménez-Morales (2021).

\section{Análisis de asociaciones de palabras}

Las unidades lexicales derivadas del estudio permiten desarrollar un análisis de coocurrencias centrado en las asociaciones de palabras, esto es, comprobar las relaciones de semejanza que determinan el significado local de los lemas dentro del corpus lingüístico. En este caso, se analiza la coocurrencia del lema más frecuente en el corpus lingüístico: sostenible.

En el gráfico 3 se observa que los lemas más próximos al lema sostenible, al centro del diagrama, presentan un mayor nivel de coocurrencias. De forma análoga, los lemas más alejados del lema central poseen menores niveles de coocurrencia. 


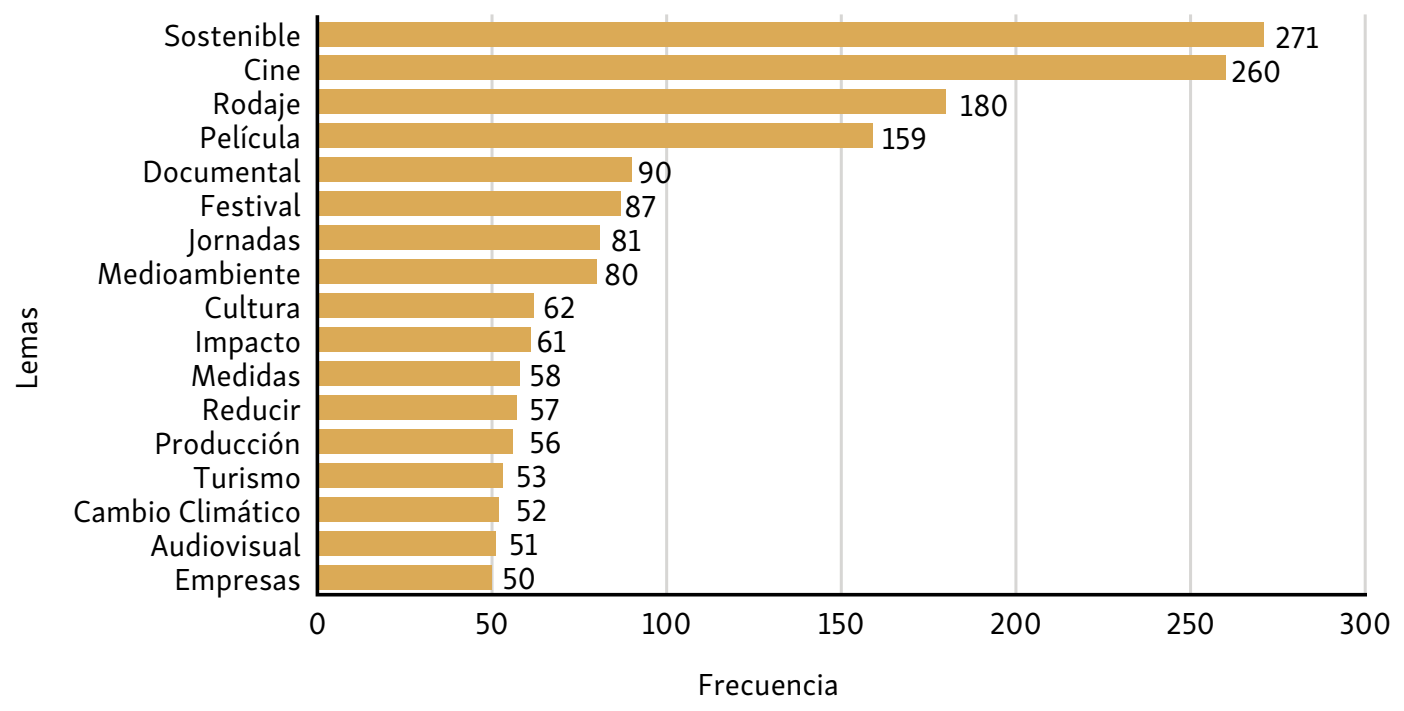

\section{Gráfico 2. Lemas más frecuentes}

Fuente: Elaboración propia.

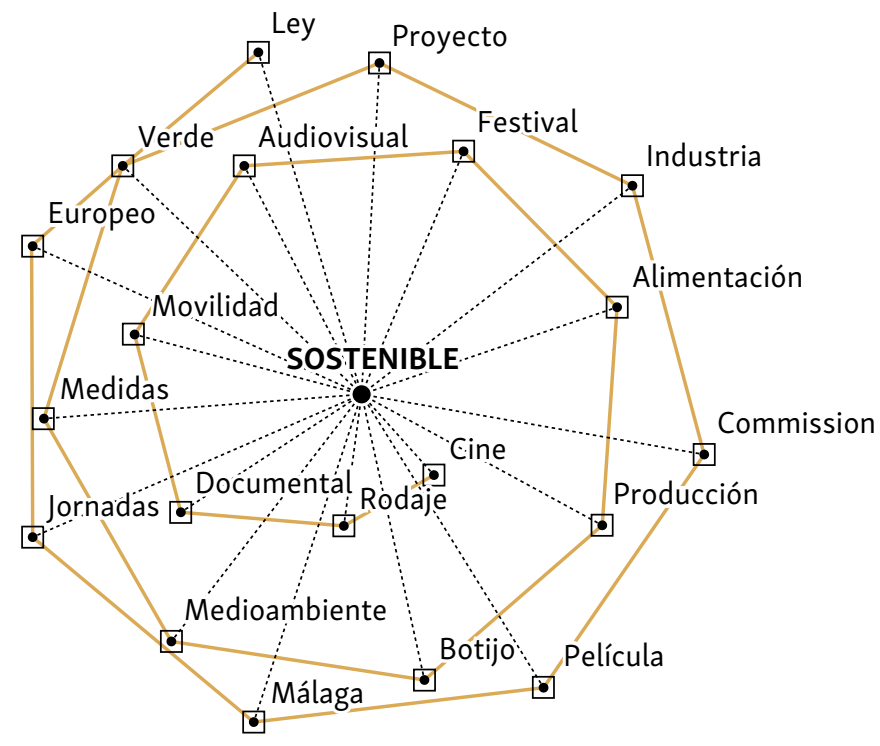

Gráfico 3. Diagrama radial de asociación de lemas para sostenible

Fuente: Elaboración propia.

De forma cuantitativa, en la tabla 2 se ofrece la asociación entre el lema sostenible con otros cuya relación es estadísticamente significativa, es decir, los lemas en los que su nivel de asociación es más fuerte con independencia de su frecuencia de repetición. Para ello, se considera el coeficiente de asociación seleccionado (Coef; coeficiente de coseno) y se muestran únicamente las coocurrencias en las que el valor de la prueba $\mathrm{Chi}^{2}$ determina un valor estadísticamente significativo $(\mathrm{p}<0,05)$. 


\begin{tabular}{|c|c|c|c|c|}
\hline LEMMA_B & COEFF & CE_AB & $\mathrm{CHI} 2$ & (p) \\
\hline Cine & 0,497 & 132 & 103,217 & 0,000 \\
\hline Rodaje & 0,407 & 90 & 60,536 & 0,000 \\
\hline Documental & 0,256 & 40 & 15,834 & 0,000 \\
\hline Movilidad & 0,239 & 18 & 38,103 & 0,000 \\
\hline Audiovisual & 0,213 & 25 & 13,629 & 0,000 \\
\hline Alimentación & 0,207 & 17 & 22,297 & 0,000 \\
\hline Botijo & 0,186 & 13 & 19,370 & 0,000 \\
\hline Green-shooting & 0,152 & 10 & 10,624 & 0,001 \\
\hline Gastronomía & 0,147 & 8 & 12,017 & 0,001 \\
\hline Cambio climático & 0,034 & 4 & 10,140 & 0,001 \\
\hline Producción & 0,203 & 25 & 9,716 & 0,002 \\
\hline Ley & 0,153 & 11 & 9,606 & 0,002 \\
\hline Commission & 0,161 & 14 & 7,965 & 0,005 \\
\hline Europeo & 0,155 & 13 & 7,381 & 0,007 \\
\hline Cultura & 0,062 & 8 & 6,443 & 0,011 \\
\hline Málaga & 0,159 & 15 & 6,110 & 0,013 \\
\hline Plástico & 0,147 & 13 & 4,995 & 0,025 \\
\hline Festival & 0,208 & 32 & 4,914 & 0,027 \\
\hline Verde & 0,168 & 19 & 4,723 & 0,030 \\
\hline Proyecto & 0,165 & 18 & 4,725 & 0,030 \\
\hline Industria & 0,163 & 18 & 4,237 & 0,040 \\
\hline Medidas & 0,175 & 22 & 3,944 & 0,047 \\
\hline Carbono & 0,134 & 11 & 3,906 & 0,048 \\
\hline Cortometrajes & 0,025 & 2 & 3,907 & 0,048 \\
\hline
\end{tabular}

Coef: coeficiente de coseno empleado como valor del índice de asociación.

$A B$ : coocurrencia en los contextos elementales de aquellos lemas $A$ : objeto de análisis (sostenible) y sus principales lemas $B$ asociados, que resultan estadísticamente significativos $(p<0,05)$.

$\mathrm{Chi}^{2}$ : estadístico que contribuye a verificar la significación de las coocurrencias.

p-valor: probabilidad de que el valor estadístico calculado Chi2 sea posible dada una hipótesis nula cierta.

El valor de significación exigida se ha fijado en $p<0,05$.

Tabla 2. Análisis de asociaciones de palabras para el lema A (sostenible)

Fuente: Elaboración propia.

Los resultados muestran que entre los lemas con mayor nivel de coocurrencia (AB) con el lema A (sostenible) destacan cine y rodaje, que son los que determinan el tema objeto de estudio. Le siguen los lemas documental, festival, audiovisual, producción y medidas. Asimismo, se constata que Málaga es el único lema que hace referencia a un territorio nacional. La región andaluza posee una dilatada 
experiencia en la producción audiovisual de carácter nacional e internacional. En los años 60 fue escenario para películas de distintos géneros, especialmente del western (Checa Godoy, 2005). Los rodajes de diversas secuencias de la quinta, sexta, séptima y octava temporada de la serie Juego de Tronos confirmaron la reputación de Andalucía como territorio acreditado para atender cualquier tipo de producción audiovisual (Andalucía Film Commission, 2018). Andalucía, y específicamente Málaga, ha sido el primer territorio español en realizar producciones audiovisuales que contemplen la sostenibilidad. La productora malagueña Fresco Film Service S.L., a cargo del rodaje en España de la serie Juego de Tronos y de Terminator: Destino oscuro ha llevado a cabo numerosas acciones orientadas a desarrollar rodajes sostenibles, como sustituir las botellas de plástico por recipientes rellenables y reutilizables.

Precisamente, esta iniciativa enlaza con el lema botijo, que también tiene una posición destacada en este análisis. Al examinar este término en el corpus lingüístico, se constata su vinculación con las noticias que abordan la implantación de la ley del botijo en los rodajes, para eliminar la utilización de miles de botellas de agua de plástico de un solo uso y desarrollar procesos de producción más sostenibles.

La tabla 3 muestra diversos contextos elementales que ilustran las asociaciones entre el lema sostenible con los lemas de mayor coocurrencia (cine, rodaje, documental, festival, audiovisual, producción y medidas). Asimismo, se incorporan contextos elementales asociando el lema sostenible con Málaga y botijo.

Madrid acoge el II Another Way Film Festival, dedicado al cine sostenible. Se proyectarán trece películas documentales en torno a la sostenibilidad social, económica y medio ambiental y actividades paralelas para niños y adultos. (La Vanguardia, 06/10/2016).

Rodajes más sostenibles con medidas como la "ley del botijo". Representantes de la industria del cine han impulsado en los últimos años distintas medidas para favorecer la sostenibilidad de los rodajes, reducir la huella ecológica de los mismos y lograr disminuir el impacto ambiental. (ABC, 28/10/2019).

Estudiantes de cine y televisión aprenden a hacer rodajes más sostenibles. Más de 90 estudiantes de diferentes disciplinas del sector audiovisual han participado este viernes en una jornada de concienciación sobre sostenibilidad y respeto al medioambiente en la que han adquirido una serie de conocimientos a aplicar en los rodajes para hacerlos más ecosostenibles. (Europa Press Andalucía, 09/11/2018).

Málaga está posicionándose como una ciudad europea de vanguardia en la promoción de los rodajes sostenibles en cine y televisión. El sector audiovisual, como tantos otros, en sus procesos de producción y distribución puede generar un impacto medioambiental que podría reducirse notablemente estableciendo estándares y políticas comunes más "verdes" y, por lo tanto, menos agresivas con nuestro entorno. (Europa Press Andalucía, 26/11/2019).

Tabla 3. Ejemplos de coocurrencias

Fuente: Elaboración propia. 


\section{Comparación entre parejas de lemas}

El siguiente examen que se ha realizado ha sido el análisis de coocurrencias centrado en la comparación entre parejas de lemas o palabras clave, lo que permite cotejar contextos elementales en los que dos lemas específicos están presentes y, de este modo, constatar los lemas más frecuentes que aparecen en la intersección de la pareja de lemas seleccionados.

En primer lugar, se comparan los lemas cine - sostenible, dado que son los que mayor relación guardan con el tema objeto de estudio y, a su vez, son los más frecuentes en el corpus lingüístico. Los resultados se muestran en histogramas (gráfico 4). En la parte izquierda se observan los lemas que coocurren para la pareja de lemas seleccionada. En la parte superior, se muestra el número de contextos elementales en los que cada lema coocurre con el término "A" (cine-color rojo), con el término "B" (sostenible-color azul) y con ambos "AB" (color verde).

Los lemas con más número de repeticiones con el lema cine (en rojo), son rodaje, jornadas, cambio climático, industria y medidas. Por su parte, la coocurrencia con el lema sostenible (en azul) se da, especialmente, con el lema rodaje. Le siguen audiovisual e impacto. Asimismo, se aprecian lemas que coocurren con la pareja de palabras clave cine - sostenible (en verde). Los principales lemas que se presentan en esta intersección son: rodaje, jornadas, industria y medidas.

Estos resultados confirman que, de forma mayoritaria, cuando las noticias abordan temas ligados al audiovisual sostenible, se centran en las medidas que la industria establece durante el período de rodaje.

Puesto que el lema rodaje ha sido el que ha presentado mayor nivel de coocurrencia en el análisis anterior, se realizó una segunda comparación, entre la pareja de lemas rodaje - sostenible, ya que resulta interesante determinar qué aspectos se destacan en cuanto a la sostenibilidad en esta etapa específica de producción.

Los lemas con más número de repeticiones con el lema rodaje (en rojo) son medidas, reducir, cine e impacto. Por su parte, la coocurrencia con el término sostenible (en azul) se da, especialmente, en el lema cine. Le siguen festival y película. Del mismo modo, se observan los lemas en los que coocurren con la pareja de lemas rodaje - sostenible. Los principales lemas presentes en esta intersección (en verde) son: cine, medidas, medioambiente, Commission, botijo y Málaga.

Se confirma, por lo tanto, que los contenidos asociados a los rodajes sostenibles se vinculan con medidas y normativas, como la ley del botijo, orientadas a reducir el impacto medioambiental de dichas producciones. Destaca el protagonismo de las Film Commission, asociaciones sin ánimo de lucro dirigidas a posicionar el territorio nacional como escenario de acogida de producciones audiovisuales (http://shootinginspain.info/). 


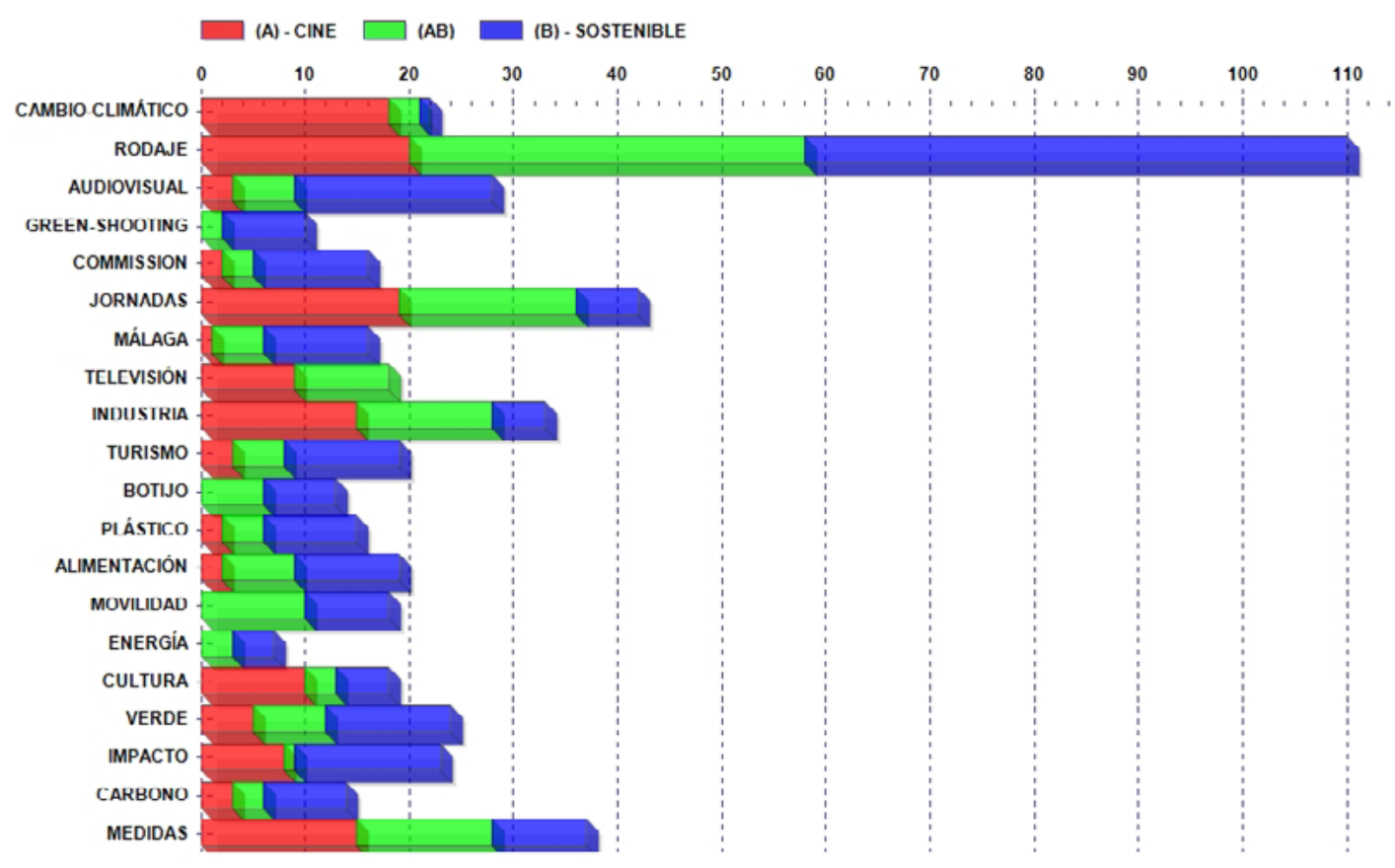

Gráfico 4. Comparación entre pareja de lemas cine - sostenible

Fuente: Elaboración propia.

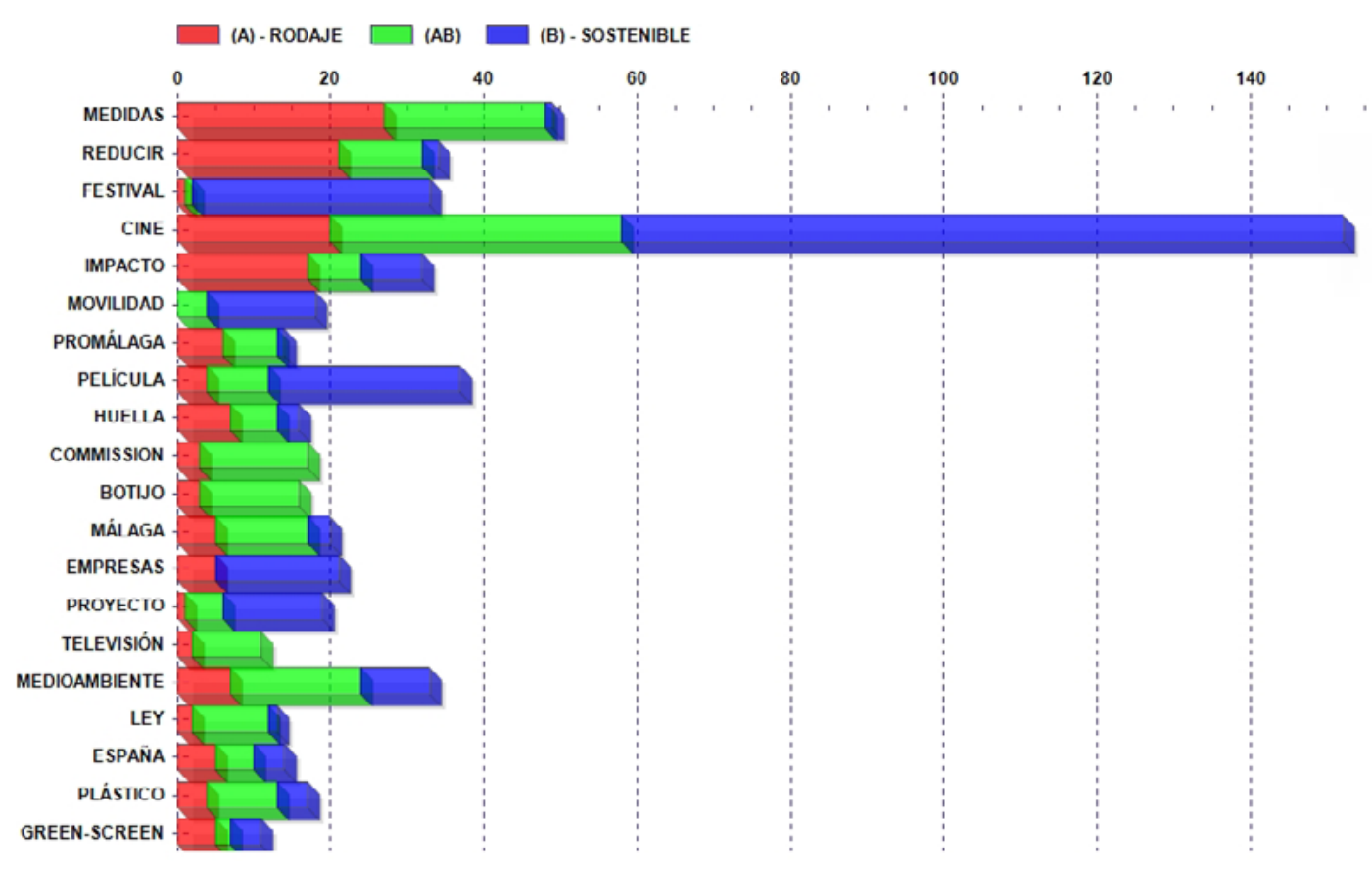

Gráfico 5. Comparación entre pareja de lemas rodaje - sostenible

Fuente: Elaboración propia. 


\begin{tabular}{ccc} 
Lemas resultantes de la intersección & Cine - sostenible & Rodaje -sostenible \\
\hline Cambio climático & +++ & \\
\hline Rodaje & ++ & \\
\hline Audiovisual & ++ & \\
\hline Green-shooting & + & +++ \\
\hline Commission & + & +++ \\
\hline Jornadas & + & +++ \\
\hline Medidas & & +++ \\
\hline Reducir & & ++ \\
\hline Festival & & ++ \\
\hline Cine & & ++ \\
\hline Impacto & &
\end{tabular}

$+0.01<$-valor $<0.05$

$++0.001<\mathrm{p}$-valor $<0.01$

+++ p-valor $<0.001$

Tabla 4. Relaciones significativas en la intersección entre pareja de lemas

Fuente: Elaboración propia.

Por otra parte, la tabla 4 muestra las relaciones estadísticamente significativas en la intersección de la comparación de las parejas de lemas cine-sostenible y rodaje-sostenible, clasificadas en función de su importancia estadística. El lema más relevante $(+++)$ derivado de la intersección entre las parejas de lemas cine - sostenible es cambio climático, mientras que para la pareja de lemas rodaje sostenible destacan los lemas medidas, reducir, festival y cine.

Los datos sugieren que la referencia noticiosa a la producción audiovisual sostenible se relaciona, principalmente, con propuestas y acciones específicas, algunas de ellas ya materializadas, para reducir el impacto medioambiental de esta industria durante el de rodaje. Asimismo, se confirma la importancia de los festivales de cine ligados a las acciones de sostenibilidad.

Nuevamente, en este análisis se constata el rol fundamental que desempeña el territorio malagueño en el diseño de iniciativas audiovisuales sostenibles. El lema Promálaga hace referencia a la empresa municipal del ayuntamiento de Málaga, que coordina rodajes sostenibles en la zona y representa a la ciudad en proyectos europeos dirigidos a impulsar la sostenibilidad de la industria audiovisual (https://www.promalaga.es/). 
Clúster 1. Propuestas reducción impacto medioambiental

\begin{tabular}{cccc} 
Lema & Clúster (Total) & $\mathbf{C h i}^{\mathbf{2}}$ & $\mathbf{p}$ \\
\hline Rodaje & $192(224)$ & 398,128 & 0,000 \\
\hline Medidas & $62(64)$ & 152,269 & 0,000 \\
\hline Botijo & $22(22)$ & 56,310 & 0,000 \\
\hline Commission & $23(30)$ & 35,066 & 0,000 \\
\hline Plástico & $23(30)$ & 35,066 & 0,000 \\
\hline Ley & $15(19)$ & 24,249 & 0,000 \\
\hline Green-shooting & $8(13)$ & 7,136 & 0,008 \\
\hline Málaga & $19(41)$ & 6,714 & 0,010 \\
\hline Reciclaje & $6(10)$ & 4,987 & 0,026 \\
\hline Reducir & $25(63)$ & 4,152 & 0,042
\end{tabular}

Clúster 3. Fomentar valores ecológicos

\begin{tabular}{cccc} 
Lema & Clúster (Total) & Chi $^{\mathbf{2}}$ & $\mathbf{p}$ \\
\hline Cultura & $63(71)$ & 216,228 & 0,000 \\
\hline Turismo & $49(64)$ & 131,693 & 0,000 \\
\hline Patrimonio & $16(16)$ & 64,628 & 0,000 \\
\hline Universidad & $26(37)$ & 59,537 & 0,000 \\
\hline Educación & $17(19)$ & 57,957 & 0,000 \\
\hline Mundial & $22(31)$ & 51,157 & 0,000 \\
\hline Jornadas & $35(68)$ & 43,455 & 0,000 \\
\hline Política & $14(18)$ & 37,975 & 0,000 \\
\hline Alimentación & $18(28)$ & 34,858 & 0,000 \\
\hline España & $20(47)$ & 15,325 & 0,000 \\
\hline Medioambiente & $28(79)$ & 12,257 & 0,000 \\
\hline Empresas & $18(47)$ & 10,100 & 0,001 \\
\hline Cambio climático & $17(44)$ & 9,796 & 0,002
\end{tabular}

\begin{tabular}{cccc} 
Lema & Clúster (Total) & Chi $^{\mathbf{2}}$ & $\mathbf{p}$ \\
\hline Europeo & $28(32)$ & 78,714 & 0,000 \\
\hline Audiovisual & $38(54)$ & 72,690 & 0,000 \\
\hline Green screen & $20(22)$ & 59,710 & 0,000 \\
\hline Huella & $28(38)$ & 58,116 & 0,000 \\
\hline Reducir & $38(63)$ & 53,138 & 0,000 \\
\hline Producción & $34(56)$ & 48,100 & 0,000 \\
\hline Carbono & $20(26)$ & 44,746 & 0,000 \\
\hline Concienciación & $20(28)$ & 38,986 & 0,000 \\
\hline Industria & $26(45)$ & 32,808 & 0,000 \\
\hline Málaga & $22(41)$ & 23,292 & 0,000 \\
\hline Promálaga & $15(25)$ & 20,436 & 0,000 \\
\hline Televisión & $13(23)$ & 15,468 & 0,000 \\
\hline Verde & $22(48)$ & 15,349 & 0,000 \\
\hline Energía & $12(22)$ & 13,124 & 0,000 \\
\hline Impacto & $25(61)$ & 12,315 & 0,000 \\
\hline
\end{tabular}

Clúster (total): número de contextos elementales que incluyen un determinado lema y, entre paréntesis, el número total de contextos elementales que incluyen ese mismo lema.

Chi²: estadístico que contribuye a verificar la significación.

p-valor: probabilidad de que el valor estadístico calculado Chi2 sea posible dada una hipótesis nula cierta. El valor de significación exigida se ha fijado en $p<0,05$.

Tabla 5. Análisis de lemas estadísticamente significativos integrados en cada clúster

Fuente: Elaboración propia.

Clúster 2. Eventos de cine sostenible

\begin{tabular}{cccc} 
Lema & Clúster(Total) & Chi $^{\mathbf{2}}$ & $\mathbf{p}$ \\
\hline Documental & $92(92)$ & 229,392 & 0,000 \\
\hline Festival & $91(92)$ & 222,388 & 0,000 \\
\hline Película & $112(113)$ & 203,195 & 0,000 \\
\hline Cine & $146(317)$ & 48,438 & 0,000 \\
\hline Gastronomía & $19(19)$ & 46,069 & 0,000 \\
\hline Cortometrajes & $22(24)$ & 45,360 & 0,000 \\
\hline Movilidad & $15(18)$ & 25,472 & 0,000 \\
\hline Sostenible & $124(331)$ & 12,000 & 0,001
\end{tabular}

Clúster 4. Tendencias del audiovisual sostenible en Europa 


\section{Análisis de clústeres}

El contenido se clasificó en grupos o clústeres temáticos significativos, mediante un método de clustering no supervisado (algoritmo bisecting k-promedios) del software T-LAB, que realiza un análisis de coocurrencias y, luego, un análisis comparativo. Se han eliminado también las unidades de contexto que no incluyan un mínimo de dos coocurrencias de los lemas seleccionados inicialmente. La partición que ha ofrecido mayor adecuación a la adherencia estadística de la muestra corresponde a cuatro clústeres.

La tabla 5 (página anterior) muestra los lemas que integran cada clúster y se identifican los siguientes ámbitos: propuestas para reducir el impacto medioambiental de estas producciones, eventos de cine sostenible, fomento de valores ecológicos y tendencias del audiovisual sostenible en Europa.

\section{Clúster 1. Propuestas para reducir el impacto medioambiental}

El clúster Propuestas para reducir el impacto medioambiental integra los lemas rodaje, medidas, botijo, Commision, plástico, ley, green-shooting, Málaga, reciclaje y reducir.

En este grupo temático todos los lemas están alineados con las iniciativas desarrolladas en la ciudad de Málaga para fomentar los rodajes sostenibles.

Por su parte, el lema Commision hace referencia a la Spain Film Commission, asociación sin ánimo de lucro que desde 2001 lidera la gestión del posicionamiento del país como destino de rodajes audiovisuales. Coordina las acciones de una amplia red de film commissions y film offices (servicios públicos de ayuntamientos) en todo el territorio español (Spain Film Commission, 2020).

A continuación, se ofrecen diversos ejemplos que ilustran este clúster (tabla 6).

En los rodajes se ha implantado "la ley del botijo" para acabar con el «sinsentido» de utilizar miles de botellas de agua de plástico de un solo uso e incluso el hecho de que cada miembro del rodaje contase con una botella reutilizable. "El botijo vuelve a estar de moda en los rodajes. Es mejor, es más barato, más ecológico y más sostenible". (Europa Press 25/10/2019).

"Nuestro interés no es solo captar rodajes, sino que se integren en el equilibrio sostenible", explicó el presidente de Spain Film Commission, Carlos Rosado, durante la firma del convenio con el Ayuntamiento de Málaga para promover las medidas de los rodajes ecológicos desarrollados desde Promálaga, en colaboración con Málaga Film Office. (Griñán, F. Diario Sur 26/11/2019).

Mallorca Film Commission trabaja para que las filmaciones sean menos agresivas con el medioambiente. Pese a la carencia de apoyo institucional, los rodajes sostenibles

(green shooting) se están introduciendo, poco a poco y con cierto recelo por parte de las productoras, en la isla. (Rodas, G. Diario de Mallorca, 06/12/2019). 


\section{Clúster 2. Eventos de cine sostenible}

El clúster 2, Eventos de cine sostenible, incorpora lemas vinculados con la celebración de festivales de exhibición de obras audiovisuales relacionadas con el desarrollo sostenible. Los principales lemas son: documental, festival, película, cortometrajes, gastronomía y movilidad.

Aquí destacan los festivales para promover los rodajes audiovisuales sostenibles e implantar criterios de sostenibilidad en todo el ámbito de la producción audiovisual, como el Festival Internacional de Cine del Medio Ambiente (FICMA), que se celebra en Barcelona desde hace 26 años. También se encuentran los festivales que se exhiben contenidos audiovisuales vinculados con el medioambiente, como el Festival Internacional de Cine Medioambiental de Canarias (FICMEC) que, en la edición de 2019, apostaba por la movilidad sostenible. La tabla 7 ilustra algunos ejemplos de este clúster.

El municipio de Garachico acogerá del 25 de mayo al 3 de junio, en el convento de San Francisco, la vigésima edición del Festival Internacional de Cine Medioambiental de Canarias (FICMEC), cuyo tema principal será la movilidad sostenible. (La Vanguardia, 15/02/2018).

El huerto de Lucas, en colaboración con Karma Films, organiza la segunda edición de

'Documentales para cambiar el planeta', un ciclo que versará sobre gastronomía ecológica y cine comprometido, que se darán cita en el patio central de El huerto durante los tres jueves de agosto. (Europa Press, 02/08/2017).

El Ayuntamiento de Las Palmas de Gran Canaria patrocina un año más el Festival Cine

+ Food. 50 películas "sostenibles". El Festival, que se celebra del 30 de agosto al 2 de septiembre, en el Parque Santa Catalina, se transforma en un evento sostenible con el ciclo Plástico Cero. (La Provincia, 09/08/2018).

\section{Tabla 7. Ejemplos de contextos elementales del clúster 2}

Fuente: Elaboración propia.

\section{Clúster 3. Fomento de valores ecológicos}

Los lemas que conforman el grupo temático Fomento de valores ecológicos presentan una gran heterogeneidad, dado que hacen referencia a diferentes sectores profesionales. Los principales lemas son cultura, turismo, universidad, educación, política, alimentación, patrimonio, jornadas y empresas.

Destacan los contenidos relativos a proyectos e iniciativas de carácter público o privado en las que las obras audiovisuales constituyen el vehículo para educar en valores orientados a preservar el medioambiente, luchar contra el cambio climático y desarrollar prácticas sostenibles en cualquier ámbito. 
Las propuestas incluidas en este clúster tienen un marcado carácter educativo, a diferencia del anterior, Eventos de cine sostenible, en el que las iniciativas, además de comprometidas, poseen cierto componente lúdico.

Algunos de los contextos elementales que describen este grupo temático se muestran en la tabla 8.

Ibicine representará a España en las rutas de cine de Movie Travel; Ibiza es una de

las ciudades españolas Patrimonio de la Unesco que formará parte de las rutas cinematográficas de Movie Travel, un proyecto financiado por la UE que apuesta por un modelo de turismo cultural sostenible en el sur de Europa con el cine como leitmotiv. El proyecto, impulsado por Inmedia Solutions, ofrece itinerarios y experiencias de viajes culturales en ciudades de Europa que son Patrimonio de la Humanidad, donde se han rodado películas o que son sede de festivales. (Nou Diari, 22/03/2019).

La Filmoteca del Institut Valencià de Cultura ha programado, con motivo de la apertura en València del Centro Mundial para la Alimentación Urbana Sostenible, un ciclo sobre la alimentación sostenible en el cine y la lucha contra modelos gastronómicos uniformes. Se trata de una selección de películas que conjuga tres pilares fundamentales de la sociedad actual como son el lenguaje audiovisual, la educación y la alimentación, y que pone de relieve cómo la alimentación sostenible está íntimamente relacionada con la defensa del territorio, la ecología, la economía y el medioambiente. (La Vanguardia, 08/05/2019).

La ETS de Ingeniería de Caminos, Canales y Puertos de la Universidad de Granada, en colaboración con Granada4Energy, y en el marco del "Programa 17 Retos: Una oportunidad para cambiar el mundo", desarrollará un ciclo de cine y debate sobre esta temática que permitirá visualizar algunas de las problemáticas que han llevado a establecer los Objetivos de Desarrollo Sostenible (ODS). (Ideal, 30/07/2019).

Tabla 8. Ejemplos de contextos elementales del clúster 3

Fuente: Elaboración propia.

\section{Clúster 4. Tendencias del audiovisual sostenible en Europa}

El clúster Tendencias del audiovisual sostenible en Europa está conformado por lemas relativos a proyectos y tendencias de la industria audiovisual, tanto en el ámbito español como en Europa: europeo, Green Screen, huella, reducir, carbono, concienciación, energía, impacto, Málaga y Promálaga.

Precisamente, en el Ayuntamiento de Málaga se integra Promálaga, orientada a la creación de empleo, la promoción empresarial y a atraer el talento que impulsa inversiones I+D y proyectos de innovación, entre otros aspectos (Promálaga, 2020). A través de esta empresa municipal y con el apoyo de la Spain Film Commision, Málaga participa en el proyecto Green Screen, orientado a identificar las políticas medioambientales a nivel europeo para implementarlas a nivel regional. De este modo, se espera compartir una guía de buenas prácticas para reducir el impacto medioambiental de las producciones audiovisuales (https://www. interregeurope.eu/greenscreen/). 
En esta misma línea, The European Producers Club - EPC (https://www. europeanproducersclub.org/), asociación formada por 130 productores independientes de cine y televisión de toda Europa, ha elaborado el Estatuto para la Producción Verde con diversos criterios para reducir el impacto medioambiental en la producción audiovisual.

La tabla 9 incluye algunos ejemplos que ilustran este clúster.

Spain Film Commission adopta el sistema de rodaje sostenible desarrollado en Málaga. La capital, única ciudad española en el programa europeo Green Screen, crea una calculadora verde para reducir la huella de carbono de las filmaciones de películas y series. (Griñán, F. Diario Sur, 26/11/2019).

El objetivo es reducir el impacto medioambiental que puedan generar los procesos de producción y distribución en los rodajes cinematográficos y publicitarios. El convenio refleja la colaboración de todas las partes para difundir entre el sector audiovisual español y extranjero este tipo de prácticas verdes que protegen el medioambiente, con campañas de concienciación y formación. (La Opinión de Málaga, 27/11/2019).

La Academia de las Artes y las Ciencias Cinematográficas de España ha anunciado este jueves 26 de diciembre su adhesión al "Estatuto para la Producción Verde, impulsado por The European Producers Club (EPC), en el que se recogen una serie de pautas para reducir el impacto medioambiental en la producción audiovisual, así como soluciones sostenibles ante las necesidades habituales de un rodaje. (La Vanguardia, 26/12/2019).

Tabla 9. Ejemplos de contextos elementales del clúster 4

Fuente: Elaboración propia.

\section{CONCLUSIONES}

La sostenibilidad es un aspecto que está generando gran interés en el sector audiovisual. Las producciones cinematográficas, televisivas y publicitarias generan un elevado nivel de contaminación. Sin embargo, esta industria es cada vez es más consciente de la necesidad de desarrollar producciones sostenibles.

España desarrolla diversas iniciativas orientadas a impulsar políticas de sostenibilidad en las producciones audiovisuales, adoptando el denominado green shooting. Aunque el tema es relativamente reciente, el análisis de la representación informativa sugiere que los medios de comunicación muestran un interés incipiente por la producción audiovisual sostenible. La presencia de este tipo de contenidos a lo largo de los cinco años del período analizado ha crecido de forma progresiva, si bien la mayor parte de las noticias publicadas procede de agencias de noticias. Su rol resulta esencial en la configuración de la opinión pública, puesto que constituyen el eslabón original en la difusión de las prácticas sostenibles del sector audiovisual y contribuyen a concientizar sobre la necesidad de desarrollar propuestas orientadas a la sostenibilidad desde sus tres vertientes: medioambiental, social y económica. 
Por otra parte, el análisis clúster del corpus lingüístico revela una agrupación temática estadísticamente significativa en torno a cuatro ámbitos: 1) propuestas para reducir el impacto medioambiental de estas producciones; 2) celebración de festivales de cine sostenible; 3) utilización de obras audiovisuales para educar en valores ecológicos, y 4) tendencias sostenibles en el sector audiovisual a nivel europeo. De este modo, el actual discurso noticioso introduce, fundamentalmente, el impacto de medidas respetuosas con el medioambiente como factores sociotécnicos de transiciones sostenibles.

Málaga destaca en los aspectos de producción audiovisual sostenible en los clústeres 1 y 4. Por una parte, como territorio precursor de iniciativas verdes para minimizar los efectos negativos de las filmaciones de películas y series y vincular a las productoras con una disciplina sostenible en su trabajo. Por otra, como ciudad europea de vanguardia en la promoción de los rodajes sostenibles en cine y televisión mediante su adhesión al programa europeo Green Screen, dirigido a diseñar políticas globales para reducir la huella de carbono, siguiendo la estrategia de implementación de los ODS para la Agenda 2030 en la categoría medioambiental.

En este sentido, se confirma que las principales soluciones adoptadas por la industria audiovisual en materia de sostenibilidad se centran en iniciativas ligadas a mitigar su impacto negativo en el entorno y evitar la degradación medioambiental. Le siguen aspectos relacionados con la educación y conscientización en torno al desarrollo sostenible, especialmente mediante festivales, jornadas y ciclos de cine. En este contexto, además de apostar por la producción sostenible de la industria audiovisual, se amplía el espectro, que se extiende al ámbito del turismo y de la alimentación, entre otros sectores.

Las implicancias gerenciales derivadas de la investigación pueden concretarse en el diseño de una guía de actuación común dirigida a optimizar los recursos y materiales de una producción, tal y como establece el decálogo de buenas prácticas del proyecto Green Screen, facilitando manuales sobre prácticas ecológicas y calculadoras de huella de carbono para conocer el impacto que genera la producción audiovisual. De este modo, se evitarían actuaciones poco respetuosas con el medioambiente. No obstante, también sería necesario configurar políticas orientadas a evitar los comportamientos sexistas y racistas originados por desigualdades de poder laboral o económico en el sector. Así pues, implementar más medidas sostenibles de carácter social podría constituir un criterio para lograr el acceso a subvenciones u otro tipo de financiamiento.

Por otra parte, la prensa debe aprovechar las medidas que aplican las empresas audiovisuales en sus producciones para generar noticias y concientizar sobre la necesidad de impulsar una industria sostenible en todos los niveles, tanto 
en el ámbito ecológico como social y laboral. Dichas informaciones tienen que desplegarse siempre desde una perspectiva de alfabetización en la sostenibilidad, y no como una estrategia comercial que redima los excesos generados durante el proceso de producción. En definitiva, resulta imprescindible impulsar estos temas en la agenda mediática para generar conciencia pública y cambios significativos en la sociedad.

\section{FINANCIAMIENTO}

Esta investigación ha sido apoyada por ESIC Business \& Marketing School (España)en el marco de su proyecto 1-V-2021. El estudio es un resultado de producción académica del Grupo de Investigación SEDDeS (Sociedad, Economía Digital y Desarrollo Sostenible).

\section{REFERENCIAS}

ABC. (2019, October 28). Rodajes más sostenibles con medidas como la "ley del botijo". . ABC. https://www.abc.es/natural/vivirenverde/abci-rodajes-mas-sostenibles-medidascomo-ley-botijo-201910271352_noticia.html

Andalucía Film Commission. (2018). Informe de Actividades (Activity Report). https://andaluciafilm.com/informes-de-actividad/

Aragón-Correa, J. A., Hurtado-Torres, N., Sharma, S., \& García-Morales, V.J. (2008). Environmental strategy and performance in small firms: A resource-based perspective. Journal of environmental management, 86(1), 88-103. https://doi.org/10.1016/j.jenvman.2006.11.022

Artero, J. P. \& Moraes, R. (2008). Opciones estratégicas de las agencias de noticias europeas: Reuters, France Presse y EFE (Strategic choice at European news agencies: Reuters, France Presse, and EFE). Comunicación y Sociedad, 21(1), 53-79. https://revistas.unav.edu/index. php/communication-and-society/article/view/36289

Barker, M. J. (2007). Global Greens and the Mass Media: Building for a Participatory Future? Griffith Journal of the Environment, 2(2), 1-21.

Brundtland, G. H. (1987). Our common future: Report of the World Commission on Environment and Development. Oxford University Press.

Carpentier, N. \& Servaes, J. (2006). Towards a Sustainable Information Society. Intellect Books.

Castelo B. M. \& Lima Rodrigues, L. (2008). Factors influencing social responsibility disclosure by Portuguese firms. Journal of Business Ethics, 83(4), 685-701.

https://doi.org/10.1007/s10551-007-9658-z

Centro de Investigaciones Sociológicas - CIS. (2019). Barómetro de noviembre 2019 (November 2019 Barometer). http://www.cis.es/cis/export/sites/default/-Archivos/ Marginales/3260_3279/3267/es3267mar.pdf 
Checa Godoy, A. (2005). Las coproducciones hispano-italianas: una panorámica (pan, amor y cine) (Spanish-Italian co-productions: an overview (bread, love, and cinema)). Padilla Libros.

Chiarini, L. \& Khedachi, N. (2019). Sustainability reporting in project-based industries: a European study with a focus on the motion picture industry (Master Thesis Malmö). https://www.diva-portal.org/smash/record.jsf?pid=diva2\%3A1483221\&dswid=175

Clevé, B. (2017). Film Production Management: How to Budget, Organize, and Successfully Shoot Your Film. Routledge.

Corbett, C. J. \& Turco, R. P. (2006). Southern California Environemntal Report Card 2006: Sustainability in the Motion Picture Industry. UCLA Institute of the Environment. https://www.ioes.ucla.edu/wp-content/uploads/mpisreport.pdf

Díaz Nosty, B. (2009). Cambio climático, consenso científico y construcción mediática. Los paradigmas de la comunicación para la sostenibilidad (Climatic change, cientific consensus and mediatic construction. The paradigm of the communication for the sustainable development). Revista Latina de Comunicación Social, (64), 99-119. https://doi.org/10.4185/RLCS-64-2009-808-99-119

Europa Press Madrid. (2017, August 2). Ocio sostenible y gastronomía 'eco' en el cine de verano de El huerto de Lucas. Europa Press. https://www.europapress.es/madrid/noticia-ociosostenible-gastronomia-eco-cine-verano-huerto-lucas-20170802142143.html

Europa Press Agro. (2019, October 25). 'La ley del botijo' y otras medidas prácticas para conseguir rodajes cinematográficos sostenibles. ('Botijo law' and other practical measures to achieve sustainable filming). Europa Press.

https://www.europapress.es/epagro/noticia-ley-botijo-otras-medidas-practicasconseguir-rodajes-cinematograficos-sostenibles-20191025192220.html

Europa Press Andalucía. (2018, November 9). Estudiantes de cine y televisión aprenden a hacer rodajes más sostenibles (Film and television students learn to make more sustainable filming). Europa Press. Retrieved from https://global.factiva.com/redir/default. aspx?P=sa\&NS=16\&AID=9UNI023600\&an=EURCU00020181109eeb9005k1\&cat=a\&ep=ASI

Europa Press Andalucía. (2019, November 26). Málaga, a la vanguardia en la promoción de los rodajes sostenibles (Malaga at the forefront in promoting sustainable filming). Europa Press. https://www.europapress.es/andalucia/malaga-00356/noticia-malaga-vanguardiapromocion-rodajes-sostenibles-20191126143248.html

Fernandes, C., Ferreira, J. J., Veiga, P. M., \& Peris-Ortiz, M. (2019). Knowledge, innovation and sustainability: past literature and future trends. In M. Peris-Ortiz, J. J. Ferreira, \& J. M. Merigó Lindahl (Eds.), Knowledge, Innovation and Sustainable Development in Organizations (pp. 11-22). Cham.

Fernández del Moral, J. \& Esteve Ramírez, F. (1993). Fundamentos de la información periodística especializada (Fundamentals of specialized journalistic information). Editorial Síntesis.

Fernández Reyes, R. (2004). Periodismo ambiental y periodismo sostenible (Environmental journalism and sustainable journalism). Ámbitos. Revista Internacional de Comunicación, (11-12), 311-317. https://revistascientificas.us.es/index.php/Ambitos/article/view/9561

Flanigan, P. (2002). The environmental cost of filmmaking. UCLA Entertainment Law Review, 10(1), 69-96. https://bit.ly/2IWOLsh 
Geels, F. W. (2010). Ontologies, socio-technical transitions (to sustainability), and the multi-level perspective. Research policy, 39(4), 495-510. https://doi.org/10.1016/j.respol.2010.01.022

Griñán F. (2019, November 26). La Spain Film Commission adopta el sistema de rodajes sostenibles desarrollados en Málaga (The Spain Film Commission adopts the sustainable filming system developed in Malaga). Diario Sur. https://www.diariosur.es/culturas/ cine/spain-film-commission-20191126151228-nt.html

Ideal. (2019, July 30). La ETS de Ingeniería de Caminos, Canales y Puertos impulsa el vínculo entre el cine y el desarrollo sostenible (The Engineering ETS of Roads, Canals and Ports promotes the link between cinema and sustainable development). Ideal. https://www.ideal.es/miugr/ ingenieria-caminos-canales-20190730111124-nt.html?ref=https\%3A\%2F\%2Fwww.google. $\mathrm{com} \% 2 \mathrm{~F}$

Johnston, J. \& Forde, S. (2011). The silent partner: News agencies and 21st century news. International Journal of Communication, 5, 195-214. https://ijoc.org/index.php/ijoc/article/view/928/519

Joyce, A. \& Paquin, R. L. (2016). The triple layered business model canvas: A tool to design more sustainable business models. Journal of cleaner production, 135, 1474-1486. https://doi.org/10.1016/j.jclepro.2016.06.067

La Opinión de Málaga. (N2019, November 27). Málaga, a la vanguardia en la promoción de los rodajes sostenibles (Malaga at the forefront in promoting sustainable filming). La Opinión de Málaga. https://www.laopiniondemalaga.es/cultura-espectaculos/2019/11/27/malagavanguardia-promocion-rodajes-sostenibles-27681845.html

La Provincia. (2018, August 9). 50 películas "sostenibles" con Cine+Food (50 "sustainable" films with Cine+Food). La Provincia. https://www.laprovincia.es/ cultura/2018/08/09/50-peliculas-sostenibles-cine-food-9441358.html

La Vanguardia. (2016, September 6). Madrid acoge el II Another Way Film Festival, dedicado al cine sostenible (Madrid hosts the II Another Way Film Festival, dedicated to sustainable cinema). La Vanguardia. https://www.lavanguardia.com/local/ madrid/20161006/41818242576/madrid-acoge-el-ii-another-way-film-festival-dedicadoal-cine-sostenible.html

La Vanguardia. (2018, February 15). El Festival de Cine Medioambiental se centra en la movilidad sostenible (The Environmental Film Festival focuses on sustainable mobility). La Vanguardia. https://www.lavanguardia.com/local/canarias/20180215/44801577117/ el-festival-de-cine-medioambiental-se-centra-en-la-movilidad-sostenible.html

La Vanguardia. (2019, May 8). La Filmoteca programa un ciclo sobre alimentación sostenible en el cine. (The filmoteca programs a cycle on sustainable food in the cinema). . La Vanguardia. https://www.lavanguardia.com/local/valencia/20190508/462126894583/lafilmoteca-programa-un-ciclo-sobre-alimentacion-sostenible-en-el-cine.html

La Vanguardia. (2019, December 23). La Academia de Cine se compromete a fomentar los rodajes sostenibles (The Film Academy is committed to promoting sustainable filming). La Vanguardia. https://www.lavanguardia.com/vida/20191226/472509513347/laacademia-de-cine-se-compromete-a-fomentar-los-rodajes-sostenibles.html

Lippman, W. (2003). La opinión pública (Public opinion). Langre. 
Lopera-Mármol, M. (2019). Green shooting y rodajes sostenibles: la nueva tendencia del sector audiovisual que ha llegado para quedarse (Green shooting and sustainable filming: the new trend in the audiovisual sector that is here to stay). Observatorio de Cibermedios (OCM). https://observatoriocibermedios.upf.edu/green-shooting

Lopera-Mármol, M. \& Jiménez-Morales, M. (2021). Green Shooting: Media Sustainability, A New Trend. Sustainability, 13(6), 3001. https://doi.org/10.3390/su13063001

Markard, J. (2012). Transformation of infrastructures: sector characteristics and implications for fundamental change. Journal of Infrastructure Systems, 17(3), 107-117. https://doi.org/10.1061/(ASCE)IS.1943-555X.0000056

Mcquail, D. (2000). Introducción a la teoría de la comunicación de masas (Mass Communication Theory. An Introduction). Paidós.

Moore, J. (2005). Barriers and pathways to creating sustainability education programs: policy, rhetoric and reality. Environmental Education Research, 11(5), 537-555. https://doi.org/10.1080/13504620500169692

Noci, G. \& Verganti, R. (1999). Managing 'green'product innovation in small firms. R\&d Management, 29(1), 3-15. https://doi.org/10.1111/1467-9310.00112

Nou Diari. (2019, March 22). Ibicine se incorpora a las rutas cinematográficas de Movie Travel (Ibicine joins Movie's cinematographic routes). Nou Diari. https://www.noudiari. es/cultura-ibiza/ibicine-se-incorpora-a-las-rutas-cinematograficas-de-movie-travel/

Núñez Ladevéze, L. (1995). Introducción al periodismo escrito (Introduction to written journalism). Ariel.

O'Brien, E. (2014). Going Green \& Saving Green: A Cost-Benefit Analysis of Sustainable Filmmaking. Producers' Guild of America. http://www.greenproductionguide.com/wp-content/ uploads/2014/05/FINAL_PGA3142014.pdf

Orlitzky, M., Siegel, D. S., \& Waldman, David A. (2011). Strategic corporate social responsibility and environmental sustainability. Business \& Society, 50(1), 6-27. https://doi.org/10.1177/0007650310394323

Rodas, G. (2019, December 6). Mallorca apostará en 2020 por los rodajes sostenibles (Mallorca will bet in 2020 on sustainable filming). Diario de Mallorca. https:// www.diariodemallorca.es/cultura/2019/12/06/mallorca-apostara-rodajessostenibles-2823124.html

Scheufele, D. A. (1999). Framing as a theory of media effects. Journal of communication, 49(1), 103-122. https://doi.org/10.1111/j.1460-2466.1999.tb02784.x

Schmidt, A., Ivanova, A., \& Schäfer, M. S. (2013). Media attention for climate change around the world: A comparative analysis of newspaper coverage in 27 countries. Global Environmental Change, 23(5), 1233-1248. https://doi.org/10.1016/j.gloenvcha.2013.07.020

Schweinsberg, S., Darcy, S., \& Cheng, M. (2017). The agenda setting power of news media in framing the future role of tourism in protected areas. Tourism Management, 62, 241-252.

Stubbs, W. \& Cocklin, C. (2008). Conceptualizing a sustainability business model. Organization E environment, 21(2), 103-127. https://doi.org/10.1177/1086026608318042 
Van Kleef, J. A. G. \& Roome, N. J. (2007). Developing capabilities and competence for sustainable business management as innovation: a research agenda. Journal of cleaner production, 15(1), 38-51. https://doi.org/10.1016/j.jclepro.2005.06.002

Victory, J. (2015). Green shoots: Environmental sustainability and contemporary film production. Studies in Arts and Humanities, 1(1), 54-68. https://doi.org/10.18193/sah.vli1.6

\section{SOBRE LA AUTORA}

VANESSA ROGER-MONZó, Doctora en Comunicación Audiovisual por la Universidad Politécnica de Valencia (2010). Licenciada en Ciencias de la Información, rama Imagen Visual y Auditiva, por el CEU San Pablo (2000), en Periodismo por la Universidad de Valencia (2005) y en Publicidad y Relaciones Públicas por la Universitat Oberta de Catalunya (2015). Ha trabajado en diversos medios de comunicación. Como investigadora, ha profundizado en la comunicación de la salud, la comunicación política de los think tanks y en el film tourism.

(iD) https://orcid.org/0000-0002-7498-0406 